\title{
Índices esclerométricos avaliados ao longo da altura da seção transversal de vigas de madeira
}

\author{
Sclerometric index evaluated along the depth of \\ cross-section of timber beams
}

\author{
Nádia Schiavon da Veiga ${ }^{{ }^{*}}$ e Julio Soriano ${ }^{1}$
}

\begin{abstract}
Resumo
As vigas de madeira apresentam a inerente variação das tensões normais decorrente da intensidade do carregamento, bem como da posição na altura da seção transversal. Nas inspeções de peças fletidas em serviço, o estado de tensões pode afetar os resultados obtidos pelos ensaios não destrutivos. Nesta pesquisa, foi avaliada a influência nos resultados de ensaios esclerométricos relativa à posição na altura de vigas de madeira. Para tal, foram empregadas cinco vigas de cumaru (Dipteryx odorata) e sete vigas de garapa (Apuleia leiocarpa), com seção transversal $60 \mathrm{~mm} \times 160 \mathrm{~mm}$ e comprimento igual a $3500 \mathrm{~mm}$. Os impactos esclerométricos foram aplicados nas faces laterais das vigas sob forças de flexão incrementais, em pontos da linha neutra teórica, bem como nas regiões de compressão e de tração. Os resultados mostraram que para a maioria das peças houve diferenciação da linha neutra, posição em que os índices esclerométricos resultaram superiores àqueles obtidos nas zonas de compressão ou de tração. Concluiu-se que a posição da linha neutra teórica é mais apropriada para aplicação dos impactos esclerométricos em peças de madeira fletidas.
\end{abstract}

Palavras-chave: Força incremental; linha neutra; ensaio não destrutivo; madeira folhosa.

\begin{abstract}
The timber beams present the inherent variation of the normal stresses due to the loading intensity, as well as of the position at the cross-section depth. In evaluation of bending beams in service, the stress state may affect the results obtained by nondestructive tests. In this research, the influence on the results of sclerometric tests relative to the position at the depth of timber beams were evaluated. For this purpose, five beams of cumaru (Dipteryx odorata) and seven beams of garapa (Apuleia leiocarpa) were used, with a cross-section of $60 \mathrm{~mm} \times 160 \mathrm{~mm}$ and a length of $3500 \mathrm{~mm}$. The sclerometric impacts were applied to the lateral faces of the beams under incremental bending forces, on points of the theoretical neutral axis, as well as at the compression and tensile zones. For most of the pieces, the results showed that there was differentiation of the neutral axis, position in which the sclerometric indexes were superior to those obtained in compression or tensile zones. It was concluded that the position of the theoretical neutral axis is more appropriate for sclerometric impacts application in timber beams.
\end{abstract}

Keywords: Incremental force; neutral axis; nondestructive test; hardwood.

\section{INTRODUÇÃO}

As forças externas aplicadas às peças estruturais podem influenciar os resultados de alguns ensaios não destrutivos (END), que são importantes ferramentas para inspeções e estimativa de propriedades físicas e mecânicas dos materiais. Baseando-se nisso, pesquisas aplicadas a diversos tipos de materiais de construção civil buscam avaliar o quanto as intensidades dos carregamentos podem afetar os resultados dos diferentes métodos END aplicados às peças estruturais em serviço.

Como exemplo de pesquisa direcionada ao estudo de correlações dos resultados de um método END em função da intensidade de carregamentos, a ultrassonografia foi aplicada a peças de concreto antes e após de serem submetidas a diferentes valores de forças de compressão (MAHMOUDABADI et al., 2014). Os autores concluíram que há uma forte correlação $(R>0,96)$ entre a intensidade de força

1. Faculdade de Engenharia Agrícola - FEAGRI, Universidade Estadual de Campinas - UNICAMP. Campinas / SP, Brasil. *Autor correspondente: nadiasveiga@gmail.com 
Veiga e Soriano - Índices esclerométricos avaliados ao longo da altura da

seção transversal de vigas de madeira

aplicada às peças e as variações na velocidade de propagação de onda ultrassônica (VPU), comprovando que o estado de tensões influencia os resultados desse método END.

O método infravermelho próximo (near infrared - NIR) foi empregado para estimativa da força aplicada em peças de madeira sob flexão a quatro pontos (ANDRÉ et al., 2006). Os autores obtiveram coeficientes de correlação $(\mathrm{R})$ superiores a 0,96 entre os resultados de NIR e as diferentes intensidades de força aplicadas às peças, tanto para a região de compressão quanto de tração.

Gonçalves e Ormonde (2005) e Hasegawa et al. (2012) avaliaram a acustoelasticidade da madeira, e concluíram que as forças de maior intensidade produzem redução na VPU, mesmo para diferentes espécies. Hasegawa et al. (2012) utilizaram corpos de prova prismáticos (60 mm x $30 \mathrm{~mm}$ x $20 \mathrm{~mm}$ ) sob compressão paralela às fibras. Gonçalves e Ormonde (2005) utilizaram vigas com seções transversais de $50 \mathrm{~mm}$ x $150 \mathrm{~mm}$ e comprimento igual a $1300 \mathrm{~mm}$, com força aplicada na direção tangencial às fibras e as leituras de tempo de propagação das ondas foram realizadas na mesma direção da força.

Faggiano et al. (2009) aplicaram os métodos END de ultrassonografia, resistográfico e esclerométrico com pino de penetração, em peças de madeira afim de se obter correlações dos resultados desses métodos com as propriedades da madeira, tais como densidade, resistência à compressão e módulo de elasticidade, obtidas por métodos de ensaio destrutivos. Os autores observaram que a esclerometria apresentou maior coeficiente de correlação entre a profundidade de penetração do pino e a densidade da madeira, para os impactos aplicados na direção normal às fibras $(R=0,55)$, enquanto que o resistógrafo se mostrou mais eficiente quando aplicado na direção longitudinal $(R=0,82)$. Pela ultrassonografia a correlação entre velocidade de onda obtida na direção tangencial e densidade resultou $\mathrm{R}=0,14$.

A esclerometria foi sugerida como método END para estimativa da densidade da madeira (SORIANO et al., 2015). Para obtenção dos índices esclerométricos os autores empregaram prismas das espécies cumaru (Dipteryx odorata), garapa (Apuleia leiocarpa) e cedro (Cedrela spp), sob força de compressão constante. Os autores concluíram que os índices esclerométricos para as três direções anatômicas da madeira se correlacionam à densidade da madeira com coeficientes de correlação superiores a 0,84 .

A análise de diferenciação nos resultados de ensaios não destrutivos quando estes são aplicados sobre a linha neutra ou nas zonas de tração e compressão se faz necessária para uma padronização de execução do método in situ. Esta pesquisa teve como objetivo avaliar a influência nos resultados da esclerometria em função da posição dos impactos aplicados ao longo da altura de vigas de madeiras folhosas submetidas à força de flexão incremental.

\section{MATERIAIS E MÉTODOS}

Foram empregadas cinco vigas da espécie cumaru (Dipteryx odorata) e outras sete vigas da espécie garapa (Apuleia leiocarpa), todas com seção transversal medindo $60 \mathrm{~mm}$ x $160 \mathrm{~mm}$ e comprimento de $3500 \mathrm{~mm}$. Com base nos procedimentos da Associação Brasileira de Normas Técnicas - ABNT NBR 7190 (ABNT, 1997) foram determinados as densidades aparentes e os teores de umidade de cada peça ensaiada. Os valores médios de densidade aparente resultaram para as espécies cumaru e garapa iguais a $1035 \mathrm{~kg} \cdot \mathrm{m}^{-3}$ e $834 \mathrm{~kg} \mathrm{~m}^{-3}$, respectivamente. Os teores de umidade médios resultaram iguais a 9,5\% para as peças de cumaru e, 9,8\% para as peças de garapa.

\section{Aplicação da esclerometria nas vigas sob força incremental de flexão}

As vigas foram submetidas a incrementos de força de flexão a quatro pontos (Fig. 01), conforme especificações da American Society for Testing and Materials - ASTM D198 (ASTM, 2014). O escopo dessa norma internacional cobre as avaliações em ensaios estáticos de peças de madeira com dimensões estruturais, diferindo, assim, da ABNT NBR 7190 (ABNT, 1997), que para a determinação da rigidez e da resistência da madeira à flexão especifica corpos de prova com seção transversal medindo $50 \mathrm{~mm}$ x $50 \mathrm{~mm}$ e o comprimento igual a $1150 \mathrm{~mm}$. Outro aspecto que foi relevante na adoção da ASTM D198 (ASTM, 2014) para esta pesquisa é o fato da força de flexão ser aplicada em dois pontos (nos terços do vão da peça estrutural), enquanto que pela norma brasileira a força é aplicada em apenas um ponto localizado no meio do vão da peça. Como consequência do esquema estático da ASTM D198 (ASTM, 2014) na região central da viga (Fig. 02) resultam momento fletor constante e força cortante nula. Assim, para cada incremento de força, esses esforços não influenciam os resultados do método END ali aplicado. 


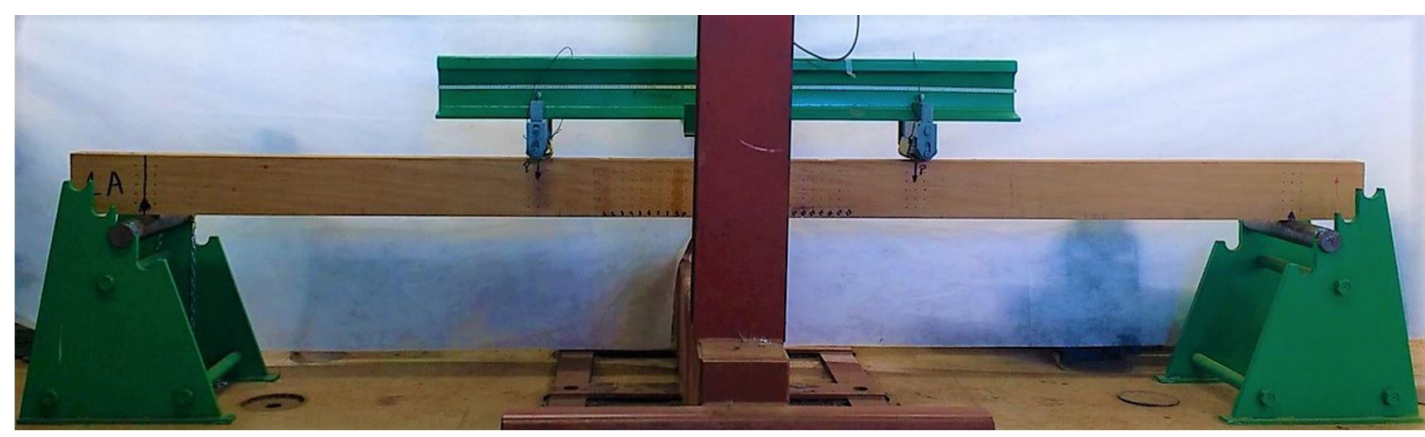

Figura 01. Viga sob flexão a quatro pontos - vista lateral durante o carregamento para a aplicação da esclerometria. Figure 01. Beam under four-point bending - side view during loading for the application of sclerometry.

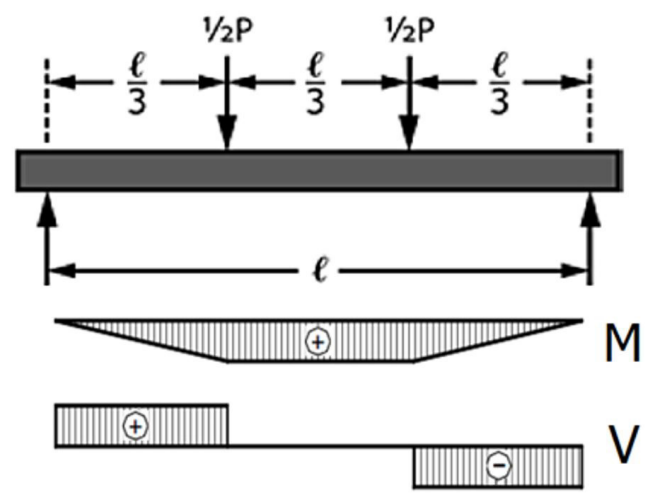

Figura 02. Esquema estatico de flexão a quatro pontos, diagramas de momento fletor e de força cortante. Figure 02. Static scheme of four-point bending, bending moment and shear force diagrams.

A distância entre apoios foi adotada igual a 20 vezes a altura das peças, ou seja, $3200 \mathrm{~mm}$. Essa dimensão foi calculada baseando-se nos princípios da norma ASTM D4761 (ASTM, 2013), que especifica a largura do vão entre apoios de 17 a 21 vezes a altura da seção transversal da peça. Por meio de um perfil metálico, a força aplicada pelo pórtico (Fig. 01) foi transmitida simetricamente em dois pontos que define o terço central do vão da viga de madeira.

Os impactos esclerométricos foram aplicados com equipamento (Digital Silver Schmidt BN, PROCEQ, Switzerland) na direção normal às fibras (ou seja, na direção da largura das vigas), em posições da região do terço central (entre os pontos de aplicação das forças). Para garantir que as forças não afetassem os resultados da esclerometria, foram desconsideradas as regiões de proximidade de $160 \mathrm{~mm}$ dos pontos de aplicações das mesmas, restando na região central da viga o comprimento de $750 \mathrm{~mm}$ onde foram demarcados os pontos para aplicação da esclerometria.

Os pontos para aplicação dos impactos esclerométricos foram distanciados entre si de no mínimo $25 \mathrm{~mm}$, conforme estabelecem as normas ASTM C805 (ASTM, 2012) e British Standards - BS EN 12504-2 (BS, 2012) elaboradas para aplicação do método esclerométrico em peças de concreto. A localização dos pontos de impactos se deu à meia altura das vigas (i.e., na posição da linha neutra teórica), bem como em duas linhas posicionadas na região comprimida e, também, sobre duas outras linhas na região tracionada (Fig. 03).

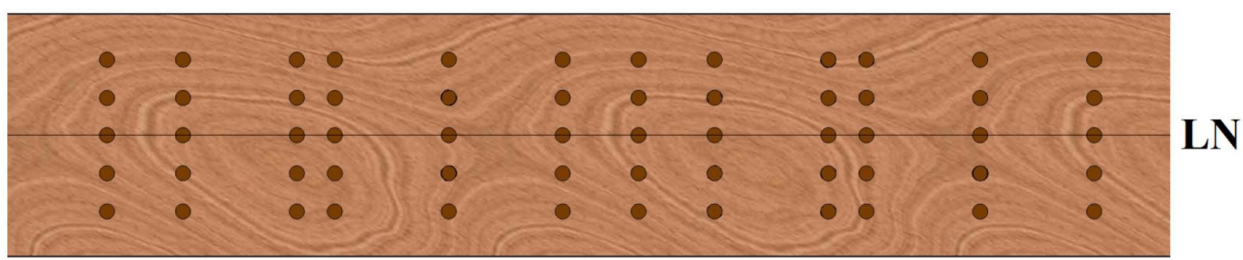

Figura 03. Pontos para aplicação da esclerometria e linha neutra teórica (LN).

Figure 03. Points for application of sclerometry and theoretical neutral axis (LN). 
Veiga e Soriano - Índices esclerométricos avaliados ao longo da altura da

seção transversal de vigas de madeira

\section{Força incremental de flexão}

Para estimar os intervalos de variação do carregamento, inicialmente foi obtido o limite de ruptura $\left(\mathrm{P}_{\lim }\right)$ para cada espécie. Para tal, duas vigas de cada espécie foram submetidas à ruptura por flexão a quatro pontos, sendo o menor valor da força de ruptura adotado como $\mathrm{P}_{\text {lim' }}$ resultando igual a $30,36 \mathrm{kN}$ para o cumaru e 45,20 kN para a garapa. Com base nesses resultados, para cada espécie foram calculados sete níveis de força para o carregamento incremental das vigas, variando entre $1,5 \mathrm{kN}\left(\mathrm{P}_{0 \%}\right)$ e $60 \%$ de $\mathrm{P}_{\lim }\left(\mathrm{P}_{60 \%}\right)$. Os impactos aplicados sob níveis de força próximos a $10 \%$ (designado como $\left.\mathrm{P}_{10 \%}\right), 30 \%\left(\mathrm{P}_{30 \%}\right)$ e $60 \%\left(\mathrm{P}_{60 \%}\right)$ da força de flexão última de cada viga (Ruptura - Tab. 01 e 02) foram utilizados para o cálculo dos índices esclerométricos.

Nota-se que as vigas C2 e C3 de cumaru (Tab. 01) romperam com forças 101\% e 57\% superiores ao valor de 30,36 kN estimado para a espécie. Como exemplo, para a viga $\mathrm{C} 2$, ao se aplicar 18,00 kN, referente a $60 \%$ da força limite de ruptura estimada para a espécie cumaru $\left(\mathrm{P}_{60 \%}\right)$, apenas $30 \%$ da força limite de ruptura real foi aplicada a essa viga, que rompeu com a força aplicada de 61,22 kN. Com isso, para as vigas C2 e C3 não foram realizados os ensaios de esclerometria nas intensidades de $60 \%$ do limite de ruptura real dessas vigas.

Tabela 01. Força de ruptura e intensidade de forças aplicadas na flexão das peças de cumaru (kN).

Table 01. Rupture force and force intensity applied in bending of cumaru beams $(\mathrm{kN})$.

\begin{tabular}{crrrrr}
\hline Intensidade de & \multicolumn{5}{c}{ Viga } \\
\cline { 2 - 6 } força & \multicolumn{1}{c}{ C1 } & C2 $^{*}$ & C3 $^{*}$ & C4 & C5 \\
\hline Ruptura & 33,64 & 61,22 & 47,80 & 27,59 & 37,23 \\
$\mathrm{P}_{10 \%}$ & 3,00 & 6,00 & 6,00 & 3,00 & 3,00 \\
$\mathrm{P}_{30 \%}$ & 12,00 & 18,00 & 15,00 & 9,00 & 12,00 \\
$\mathrm{P}_{60 \%}$ & 18,00 & - & - & 18,00 & 18,00 \\
\hline
\end{tabular}

*vigas com força de ruptura superior à força estimada para o grupo dessa espécie, por isso a esclerometria não foi aplicada à intensidade de força de $60 \%$. *beams with rupture force higher than the estimated force for the group of this species, therefore, sclerometry was not applied at $60 \%$ force intensity.

No caso da espécie garapa, algumas vigas romperam com forças inferiores àquela estimada para a espécie ( $\left.\mathrm{P}_{\lim }=45,20 \mathrm{kN}\right)$, como é o caso da viga G7, que rompeu sob a força aplicada de 22,57 kN, o que representa $49,9 \%$ de $\mathrm{P}_{\lim }$ (Tab. 02). Com isso, ao se aplicar os níveis de carregamento calculados com a força estimada, as forças aplicadas à viga G7 ficaram superestimadas, assim, o nível $\mathrm{P}_{60 \%}$ foi alcançado com apenas $13,5 \mathrm{kN}$ ( $30 \%$ do valor $\mathrm{P}_{\lim }$ para a espécie). O mesmo ocorreu para as vigas G3 e G5, que romperam a $62,3 \%$ e $64,1 \%$ da força de ruptura estimada para a espécie, respectivamente.

Tabela 02. Força de ruptura e intensidade de forças aplicadas na flexão às peças de garapa $(\mathrm{kN})$.

Table 02. Rupture force and force intensity applied in bending of garapa beams $(\mathrm{kN})$.

\begin{tabular}{crrrrrrr}
\hline Intensidade & \multicolumn{7}{c}{ Viga } \\
\cline { 2 - 8 } de força 1 & \multicolumn{1}{c}{ G1 } & G2 & \multicolumn{1}{c}{ G3 } & G4 & \multicolumn{1}{c}{ G5 } & \multicolumn{1}{c}{ G6 } & G7 \\
\hline Ruptura & 43,63 & 40,71 & 28,14 & 47,98 & 28,98 & 49,75 & 22,57 \\
$\mathrm{P}_{10 \%}$ & 4,50 & 4,50 & 1,50 & 4,50 & 1,50 & 4,50 & 1,50 \\
$\mathrm{P}_{30 \%}$ & 13,50 & 13,50 & 9,00 & 13,50 & 9,00 & 13,50 & 9,00 \\
$\mathrm{P}_{60 \%}$ & 27,00 & 27,00 & 18,00 & 27,00 & 18,00 & 27,00 & 13,50 \\
\hline
\end{tabular}

Para cada intensidade de força foi aplicado um impacto esclerométrico (Fig. 04) a cada ponto de cada coluna (Fig. 05), com repetição para os pontos da correspondente coluna da face oposta. Esse procedimento foi repetido em quatro seções 'sistematicamente alternadas e espaçadas' no trecho central da viga (Fig. 05). Assim, foram aplicados oito impactos por linha horizontal, totalizando 40 impactos para cada estágio do carregamento. 


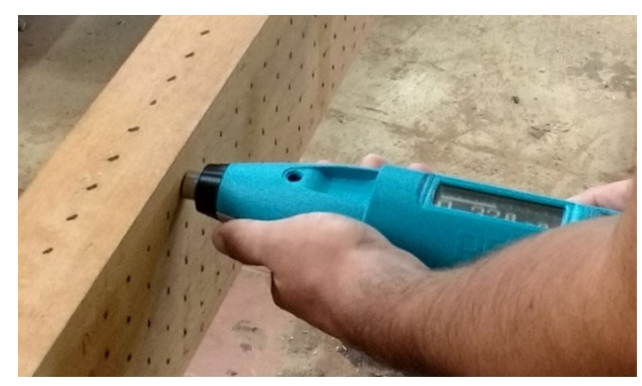

Figura 04. Impacto esclerométrico.

Figure 04. Sclerometric impact.

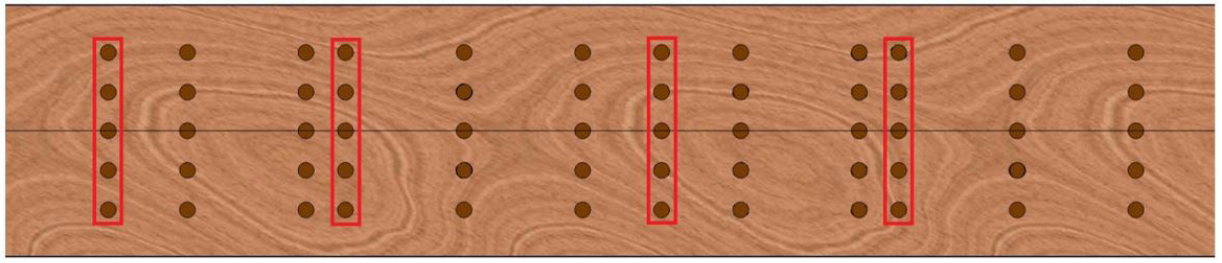

(a)

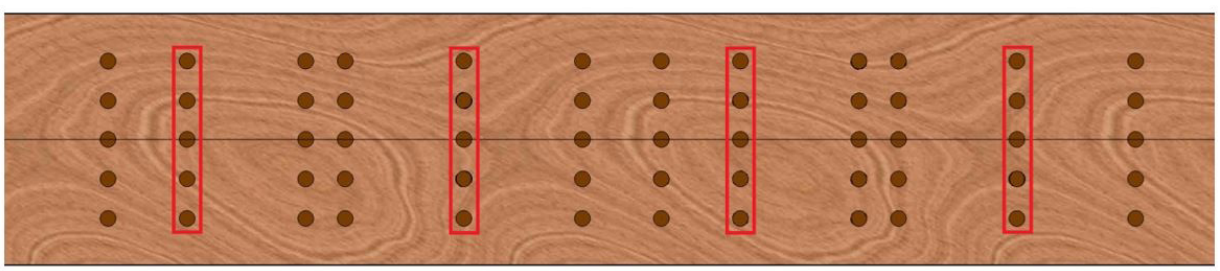

(b)

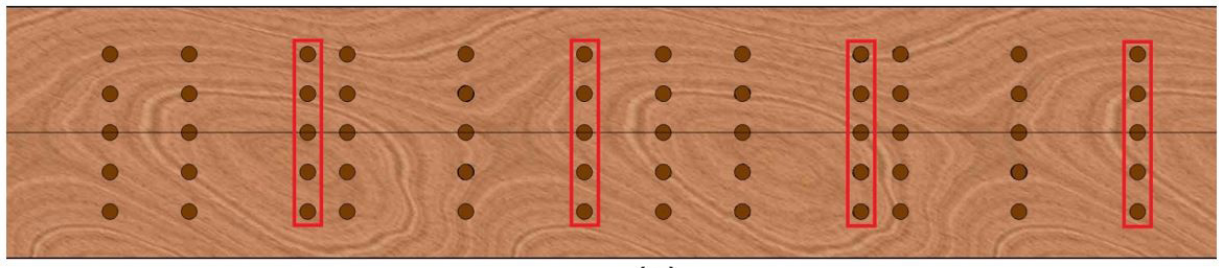

(c)

Figura 05. Colunas de pontos de aplicação da esclerometria para os três incrementos de força: (a) 10\%, (b) 30\%, (c) $60 \%$ da força de ruptura.

Figure 05. Columns of sclerometry application points for the three force increments: (a) $10 \%$, (b) $30 \%$, (c) $60 \%$ of the rupture force.

\section{Cenários para análise dos impactos esclerométricos}

Para avaliação dos impactos esclerométricos foram considerados três cenários, que levam em conta o posicionamento dos pontos de impactos na altura da viga. No primeiro cenário, considerou-se o agrupamento dos pontos aplicados sobre a linha neutra teórica (LN) e mais os pontos da linha acima e da linha abaixo da LN. Esses pontos foram agrupados numa região próxima a LN e denominada de região N (Fig. 06a). Com a análise estatística, os dados dos impactos dessa região $\mathrm{N}$ foram avaliados em relação aos grupos de impactos obtidos sobre as linhas mais afastadas da linha LN, ou seja, na região de compressão (C) e na região de tração (T). Para cada nível de força foram aplicados os impactos esclerométricos em quatro seções (quatro colunas com três pontos por face), totalizando-se em cada viga 24 impactos na região $(\mathrm{N})$ e, oito impactos em cada uma das regiões (C) e (T).

No segundo cenário (Fig. 06b) foi isolada a linha neutra teórica (LN). As duas linhas da parte superior foram agrupadas para geração dos índices esclerométricos da zona de compressão (C) e, as duas linhas da região inferior agrupadas para o cálculo dos índices esclerométricos da zona de tração (T). Com isso, no total, a cada nível de força foram aplicados oito impactos esclerométricos sobre a linha (N) e 16 impactos em cada uma das regiões de tração e de compressão. 
Veiga e Soriano - Índices esclerométricos avaliados ao longo da altura da

seção transversal de vigas de madeira

No terceiro cenário foram considerados os pontos sobre a linha neutra teórica (LN) e os pontos das linhas mais externas e posicionadas nas regiões (C) e (T). Neste cenário buscou-se avaliar a relação dos resultados da esclerometria com a forma conhecida do diagrama teórico de tensões normais e, também, avaliar a influência da proximidade às bordas nos resultados da esclerometria. Cada índice esclerométrico foi então calculado com a média dos valores dos impactos registrados sobre cada uma das três linhas (Fig. 06c). Com isso, a cada nível de força foram aplicados oito impactos esclerométricos sobre cada uma das linhas ( $\mathrm{N}, \mathrm{C}$ e T).

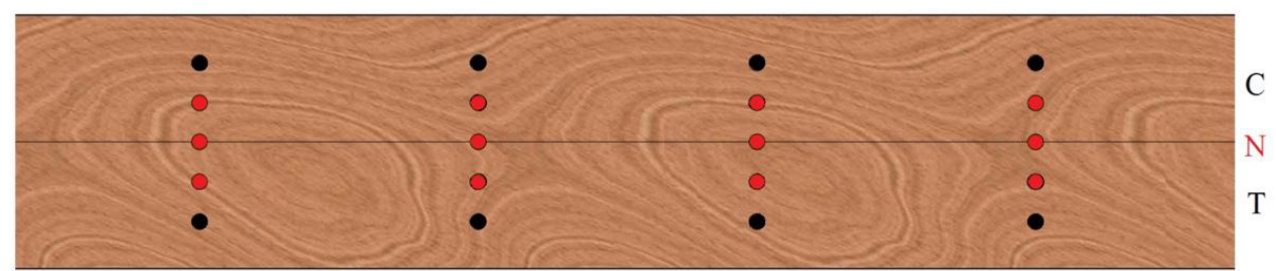

(a)

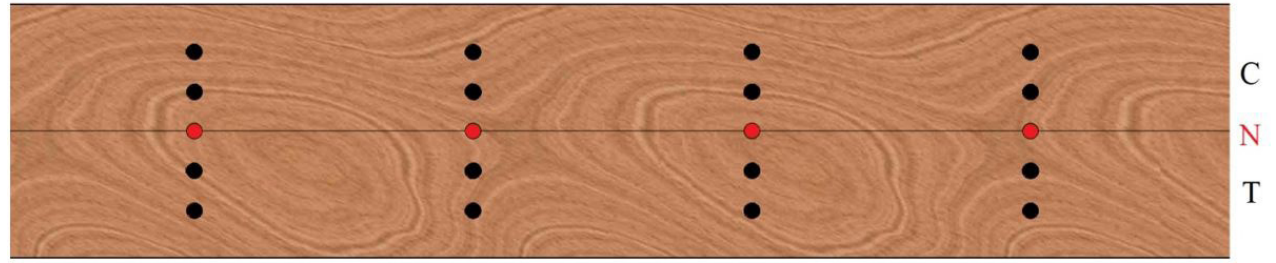

(b)

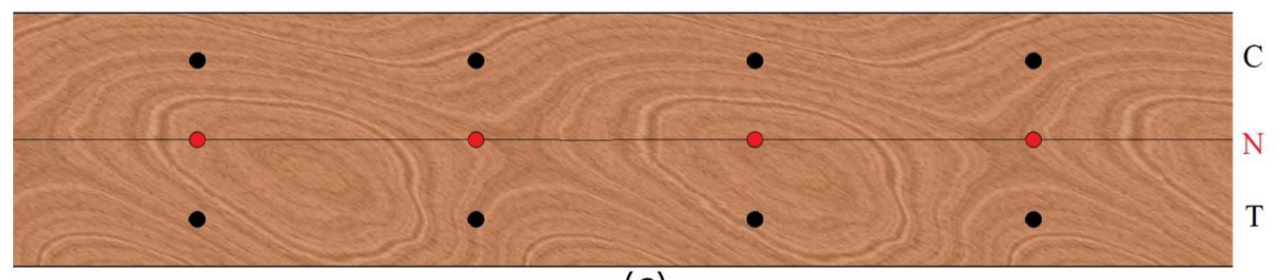

(c)

Figura 06. Pontos de aplicação dos impactos esclerométricos em uma face da viga e regiões para análise estatística: (a) primeiro cenário, (b) segundo cenário, (c) terceiro cenário.

Figure 06. Points of application of the sclerometric impacts on a beam face and regions for statistical analysis: (a) first scenario, (b) second scenario, (c) third scenario.

\section{Análise Estatística}

Os índices esclerométricos (IE) foram obtidos do cálculo das médias dos impactos esclerométricos (Q), cuja normalidade de dados foi avaliada por assimetria e curtose. Os agrupamentos foram elaborados por ANOVA a 95\% de confiabilidade, pelo método de Tukey com 5\% de erro. Essas análises foram realizadas com o programa estatístico Minitab 16 (Minitab, Canadá, 2010).

\section{RESULTADOS E DISCUSSÃO}

A análise estatística foi realizada para cada espécie, para os valores de impactos esclerométricos obtidos nos dois primeiros cenários. Pela média dos impactos esclerométricos obteve-se os índices esclerométricos, e através de valores de assimetria e curtose entre -2,0 e 2,0, comprovou-se a normalidade dos dados, com índices esclerométricos e demais análises apresentadas nas Tabelas 03 a 06 . Para análise pelo terceiro cenário utilizou-se elementos dos cenários anteriores, não sendo necessária a repetição das análises dos mesmos.

\section{Vigas da espécie Cumaru}

A análise por agrupamento dos índices esclerométricos (Fig. 07) obtidos para a espécie de maior densidade (cumaru), a cada intensidade de força aplicada, mostrou para o primeiro cenário que os resultados da esclerometria não se distinguiram em sua plenitude quanto aos impactos aplicados em pontos posicionados nas regiões (C), (N) e (T). A análise estatística apresentou igualdade (letras iguais para regiões distintas) de resultados mesmo com o aumento da força que causa flexão a quatro 
pontos. Isto mostra que os resultados da esclerometria não foram afetados diretamente pelo estado de tensões da viga.

Pelo primeiro cenário (Fig. 07), casos em que os resultados apresentaram diferenças estatísticas para os pontos localizados na região $(\mathrm{N})$ em relação às regiões $\mathrm{C}$ e $\mathrm{T}$ foram apenas registrados para a viga C2 com a intensidade de força igual a $\mathrm{P}_{10 \%}$, para a viga C4 com $\mathrm{P}_{60 \%}$ e para a viga C5 com $\mathrm{P}_{10 \%}$. Nos outros casos, os resultados ficaram compartilhados entre dois grupos, a exemplo das vigas C1, C2 e C3 $\left(\mathrm{P}_{30 \%}\right)$, não permitindo, portanto, a diferenciação pela análise estatística.

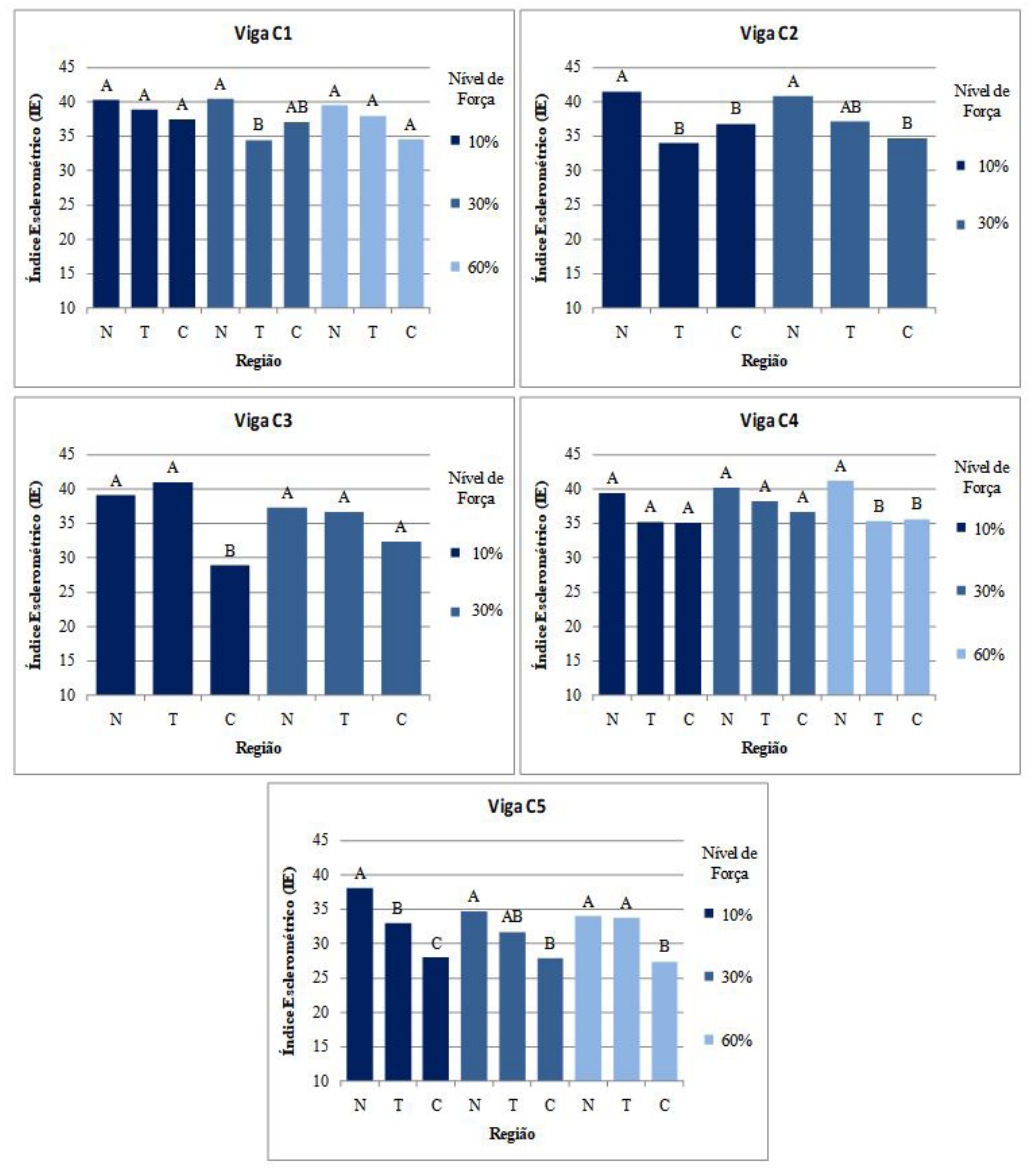

Figura 07. Índices esclerométricos por região de aplicação dos impactos às vigas de cumaru agrupados conforme o primeiro cenário.

Figure 07. Sclerometric indexes by zone of application of impacts to cumaru beams grouped according to the first scenario.

Tabela 03. Índices esclerométricos e análise estatística obtidos para as vigas de cumaru pelo primeiro cenário.

Table 03. Sclerometric indexes and statistical analysis obtained for cumaru beams by the first scenario.

\begin{tabular}{|c|c|c|c|c|c|c|c|c|c|c|}
\hline \multirow{2}{*}{\multicolumn{2}{|c|}{ Viga Análise . }} & \multicolumn{3}{|c|}{$\mathbf{P}_{10 \%}$} & \multicolumn{3}{|c|}{$\mathbf{P}_{30 \%}$} & \multicolumn{3}{|c|}{$P_{60 \%}$} \\
\hline & & C & $\mathbf{N}$ & $T$ & C & $\mathbf{N}$ & $T$ & C & $\mathbf{N}$ & $T$ \\
\hline \multirow[t]{5}{*}{ C1 } & $\mathrm{IE}^{(1)}$ & 37,4 & 40,3 & 38,9 & 37,1 & 40,4 & 34,4 & 34,6 & 39,5 & 38,0 \\
\hline & $\mathrm{DP}^{(2)}$ & 6,1 & 5,0 & 4,9 & 6,2 & 4,3 & 3,8 & 5,7 & 5,5 & 5,9 \\
\hline & $\mathrm{CV}^{(3)}$ & 16,2 & 12,3 & 12,7 & 16,7 & 10,7 & 11,0 & 16,5 & 14,0 & 15,6 \\
\hline & $\mathrm{A}^{(4)}$ & $-1,08$ & 0,11 & $-0,77$ & $-0,43$ & $-0,10$ & 0,61 & 0,05 & $-0,03$ & $-0,63$ \\
\hline & $C^{(5)}$ & 0,09 & $-1,03$ & $-1,57$ & $-1,23$ & $-1,12$ & $-1,88$ & $-1,01$ & $-1,20$ & 0,39 \\
\hline \multirow[t]{5}{*}{ C2 } & $\mathrm{IE}$ & 36,8 & 41,5 & 34,0 & 34,6 & 40,8 & 37,1 & & Rompeu (6) & \\
\hline & DP & 3,7 & 4,0 & 3,4 & 4,5 & 4,5 & 4,5 & & & \\
\hline & cV & 10,1 & 9,7 & 10,1 & 12,9 & 11,1 & 12,2 & & & \\
\hline & A & $-1,16$ & $-0,23$ & $-0,50$ & $-0,42$ & 0,18 & $-0,97$ & & & \\
\hline & C & 1,16 & 0,36 & $-0,04$ & $-1,92$ & $-1,01$ & $-0,57$ & & & \\
\hline
\end{tabular}

(I) Índice esclerométrico (2) Desvio padrão (3) Coeficiente de variação (4) Assimetria (5) Curtose (6) As vigas romperam antes que a força estimada fosse alcançada, assim, não foram realizados ensaios esclerométricos ao nível de $60 \%$ da força aplicada.

(I) Sclerometric index (2) Standard deviation (3) Coefficient of variation (4) Skewness (5) Kurtosis (6) The rupture of the beams occurred before the estimated force was reached, therefore, sclerometric tests were not performed at the level of $60 \%$ of the force applied. 
Veiga e Soriano - Índices esclerométricos avaliados ao longo da altura da

seção transversal de vigas de madeira

Tabela 03. Continuação...

Table 03. Continued..

\begin{tabular}{|c|c|c|c|c|c|c|c|c|c|c|}
\hline \multirow{2}{*}{\multicolumn{2}{|c|}{ Viga Análise . }} & \multicolumn{3}{|c|}{$\mathbf{P}_{10 \%}$} & \multicolumn{3}{|c|}{$\mathbf{P}_{30 \%}$} & \multicolumn{3}{|c|}{$\mathbf{P}_{60 \%}$} \\
\hline & & C & $\mathbf{N}$ & $\mathrm{T}$ & C & $\mathbf{N}$ & $\mathbf{T}$ & C & $\mathbf{N}$ & $T$ \\
\hline \multirow[t]{5}{*}{ C3 } & IE & 28,9 & 39,1 & 36,1 & 32,4 & 37,3 & 36,6 & & Rompeu ${ }^{(6)}$ & \\
\hline & DP & 3,5 & 4,3 & 4,6 & 5,1 & 5,6 & 5,6 & & & \\
\hline & CV & 12,1 & 11,1 & 12,8 & 15,9 & 14,9 & 15,2 & & & \\
\hline & $A$ & 1,16 & $-0,03$ & $-0,92$ & 0,17 & 0,04 & $-0,92$ & & & \\
\hline & $\mathrm{C}$ & 0,62 & $-0,42$ & $-1,51$ & $-1,52$ & $-0,65$ & $-0,98$ & & & \\
\hline \multirow[t]{5}{*}{ C4 } & IE & 35,0 & 39,4 & 35,2 & 36,6 & 40,2 & 38,2 & 35,6 & 41,3 & 35,4 \\
\hline & DP & 5,3 & 4,3 & 5,5 & 6,7 & 4,6 & 1,2 & 3,9 & 4,5 & 5,2 \\
\hline & CV & 15,1 & 11,0 & 15,7 & 18,3 & 11,5 & 3,2 & 11,0 & 10,8 & 14,8 \\
\hline & $A$ & 0,00 & 0,29 & $-0,82$ & $-0,33$ & $-0,01$ & $-0,31$ & $-0,16$ & $-0,11$ & $-0,08$ \\
\hline & C & $-0,90$ & $-1,03$ & $-0,93$ & $-1,82$ & $-1,29$ & $-1,83$ & $-1,81$ & $-1,04$ & $-1,28$ \\
\hline \multirow[t]{5}{*}{ C5 } & IE & 27,9 & 38,0 & 32,9 & 27,8 & 34,7 & 31,7 & 27,4 & 34,0 & 33,8 \\
\hline & DP & 4,2 & 3,8 & 4,0 & 6,1 & 4,2 & 5,7 & 4,7 & 5,0 & 1,9 \\
\hline & CV & 15,1 & 10,1 & 12,2 & 22,0 & 12,0 & 18,0 & 17,1 & 14,7 & 5,5 \\
\hline & A & 0,14 & $-0,43$ & $-1,11$ & 0,91 & 0,14 & $-0,07$ & $-0,37$ & $-0,30$ & 0,49 \\
\hline & C & $-1,30$ & $-0,46$ & 1,15 & $-0,40$ & $-1,07$ & $-0,88$ & $-1,33$ & $-0,86$ & $-1,55$ \\
\hline
\end{tabular}

(I) Índice esclerométrico (2) Desvio padrão (3) Coeficiente de variação (4) Assimetria (5) Curtose (6) As vigas romperam antes que a força estimada fosse alcançada, assim, não foram realizados ensaios esclerométricos ao nível de $60 \%$ da força aplicada.

(I) Sclerometric index (2) Standard deviation (3) Coefficient of variation (4) Skewness (5) Kurtosis (6) The rupture of the beams occurred before the estimated force was reached, therefore, sclerometric tests were not performed at the level of $60 \%$ of the force applied.

No segundo cenário (Fig. 08), deixam de existir as diferenciações observadas no primeiro cenário para as vigas C1 $\left(\mathrm{P}_{30 \%}\right), \mathrm{C} 2\left(\mathrm{P}_{10 \%}\right.$ e $\left.\mathrm{P}_{30 \%}\right)$, C3 $\left(\mathrm{P}_{10 \%}\right)$, C4 $\left(\mathrm{P}_{60 \%}\right)$ e C5 $\left(\mathrm{P}_{10 \%}\right.$ e $\left.\mathrm{P}_{30 \%}\right)$, implicando em grupos homogêneos ou grupos nos quais os valores não se distinguem plenamente. Para as demais vigas e carregamentos, de maneira análoga ao primeiro cenário, não houve distinção dos impactos esclerométricos em virtude das regiões em que esses foram aplicados.

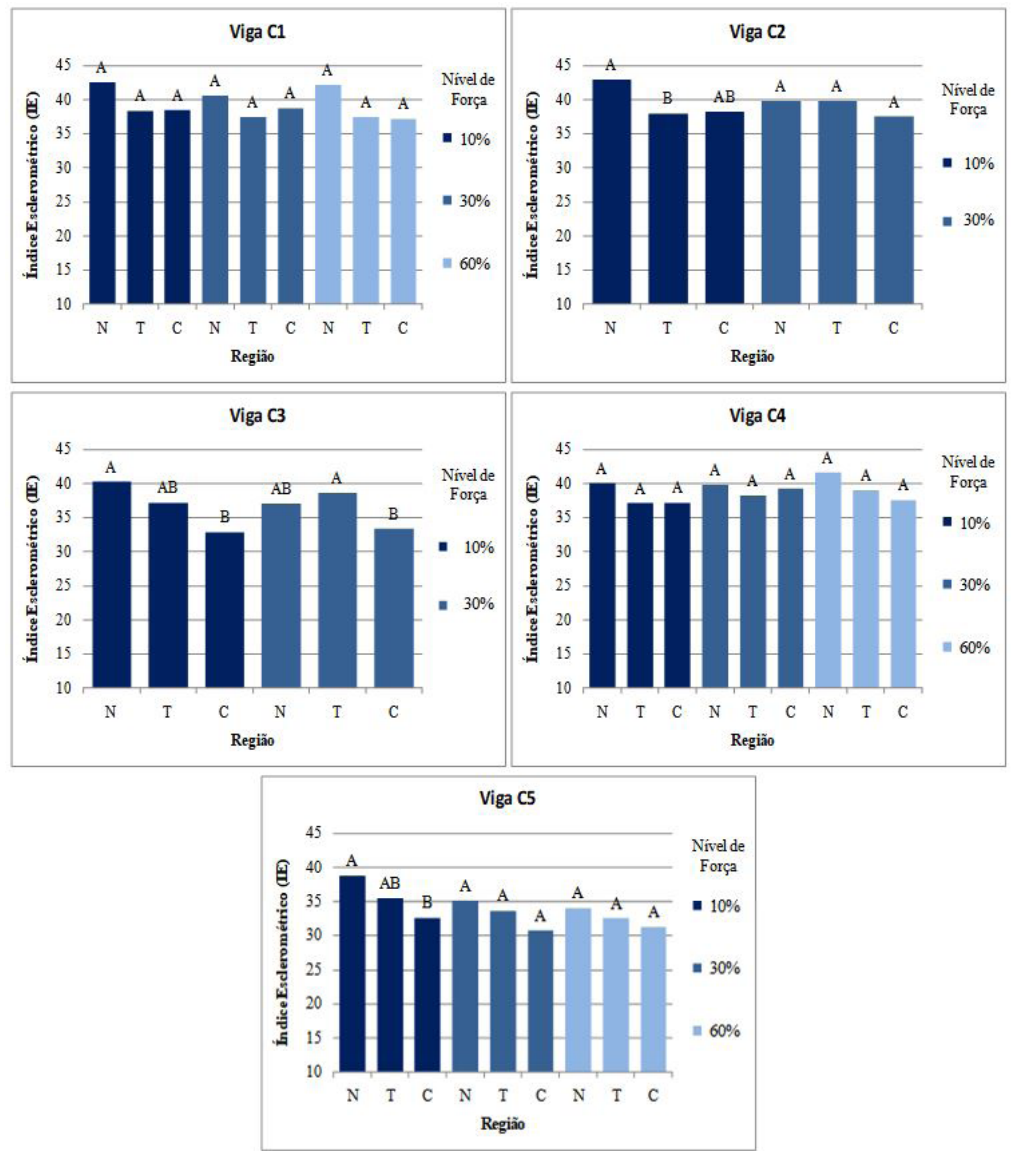

Figura 08. Índices esclerométricos por região de aplicação dos impactos às vigas de cumaru agrupados conforme o segundo cenário.

Figure 08. Sclerometric indexes by zone of application of impacts to cumaru beams grouped according to the second scenario. 
Tabela 04. Índices esclerométricos e análise estatística obtidos para as vigas de cumaru pelo segundo cenário.

Table 04. Sclerometric indexes and statistical analysis obtained for cumaru beams by the second scenario.

\begin{tabular}{|c|c|c|c|c|c|c|c|c|c|c|}
\hline \multirow{2}{*}{\multicolumn{2}{|c|}{ Viga Análise . }} & \multicolumn{3}{|c|}{$\mathbf{P}_{10 \%}$} & \multicolumn{3}{|c|}{$\mathbf{P}_{30 \%}$} & \multicolumn{3}{|c|}{$\mathbf{P}_{60 \%}$} \\
\hline & & C & $\mathbf{N}$ & $T$ & $\mathrm{C}$ & $\mathbf{N}$ & $T$ & C & $\mathbf{N}$ & $T$ \\
\hline \multirow[t]{5}{*}{$\mathrm{C} 1$} & $\mathrm{IE}^{(1)}$ & 38,5 & 42,5 & 38,4 & 38,8 & 40,6 & 37,5 & 37,2 & 42,1 & 37,4 \\
\hline & $\mathrm{DP}^{(2)}$ & 5,4 & 4,0 & 5,4 & 5,3 & 5,4 & 4,9 & 6,2 & 4,5 & 5,6 \\
\hline & $\mathrm{CV}^{(3)}$ & 14,0 & 9,4 & 14,0 & 13,7 & 13,4 & 13,0 & 16,6 & 10,6 & 14,9 \\
\hline & $\mathrm{A}^{(4)}$ & $-0,67$ & $-0,01$ & 0,28 & $-0,67$ & $-0,12$ & 0,07 & 0,08 & $-0,44$ & $-0,06$ \\
\hline & $C^{(5)}$ & 0,27 & $-0,38$ & $-0,86$ & $-0,01$ & $-1,72$ & $-1,25$ & $-0,79$ & $-0,44$ & $-0,56$ \\
\hline \multirow[t]{5}{*}{$\mathrm{C} 2$} & $\mathrm{IE}$ & 38,2 & 42,9 & 38,0 & 37,5 & 39,8 & 39,7 & & Rompeu ${ }^{(6)}$ & \\
\hline & DP & 4,0 & 3,7 & 5,6 & 4,8 & 3,9 & 5,9 & & & \\
\hline & CV & 10,4 & 8,6 & 14,6 & 12,7 & 9,8 & 14,8 & & & \\
\hline & A & $-0,85$ & 0,25 & 0,21 & $-0,57$ & $-0,07$ & 0,03 & & & \\
\hline & C & 0,45 & $-1,17$ & $-0,35$ & $-0,18$ & $-1,04$ & $-0,75$ & & & \\
\hline \multirow[t]{5}{*}{ C3 } & IE & 32,9 & 40,3 & 37,2 & 33,3 & 37,0 & 38,6 & & Rompeu (6) & \\
\hline & DP & 5,4 & 5,2 & 5,5 & 5,0 & 5,8 & 5,3 & & & \\
\hline & CV & 16,4 & 12,9 & 14,7 & 15,0 & 15,5 & 13,8 & & & \\
\hline & $A$ & 0,03 & $-0,36$ & $-0,40$ & $-0,04$ & 0,40 & $-0,54$ & & & \\
\hline & C & $-1,67$ & $-1,50$ & $-0,33$ & $-1,43$ & $-1,56$ & 0,42 & & & \\
\hline \multirow[t]{5}{*}{ C4 } & IE & 37,2 & 40,1 & 37,1 & 39,2 & 39,8 & 38,2 & 37,6 & 41,6 & 39,0 \\
\hline & DP & 4,8 & 4,7 & 5,6 & 6,4 & 5,0 & 3,5 & 4,9 & 4,3 & 5,8 \\
\hline & $\mathrm{CV}$ & 12,8 & 11,8 & 15,0 & 16,3 & 12,5 & 9,2 & 13,0 & 10,3 & 14,8 \\
\hline & $A$ & $-0,61$ & 0,47 & $-0,14$ & $-0,57$ & 0,29 & $-0,59$ & 0,62 & $-0,34$ & $-0,47$ \\
\hline & C & $-0,22$ & $-0,79$ & $-0,26$ & $-0,80$ & $-1,58$ & 0,62 & 0,65 & $-1,82$ & $-0,62$ \\
\hline \multirow[t]{5}{*}{ C5 } & IE & 32,6 & 38,7 & 35,5 & 30,7 & 35,0 & 33,6 & 31,3 & 34,1 & 32,5 \\
\hline & DP & 6,0 & 4,2 & 5,0 & 5,6 & 5,1 & 5,3 & 6,3 & 3,9 & 5,0 \\
\hline & CV & 18,3 & 10,9 & 14,0 & 18,2 & 14,5 & 15,6 & 20,3 & 11,3 & 15,3 \\
\hline & $A$ & $-0,36$ & $-0,79$ & $-0,19$ & $-0,22$ & 0,18 & $-0,41$ & 0,07 & $-0,92$ & $-0,45$ \\
\hline & C & $-1,14$ & 0,60 & 0,05 & $-1,12$ & $-1,21$ & $-0,73$ & $-0,64$ & 1,28 & $-0,31$ \\
\hline
\end{tabular}

(I) Índice esclerométrico; (2) Desvio padrão; (3) Coeficiente de variação; (4) Assimetria; (5) Curtose; (6) As vigas romperam antes que a força estimada fosse alcançada, assim, não foram realizados ensaios esclerométricos ao nível de $60 \%$ da força aplicada.

(I) Sclerometric index (2) Standard deviation (3) Coefficient of variation (4) Skewness (5) Kurtosis (6) The rupture of the beams occurred before the estimated force was reached, therefore, sclerometric tests were not performed at the level of $60 \%$ of the force applied.

Analogamente aos resultados do primeiro e do segundo cenário, no terceiro cenário os índices esclerométricos das vigas de cumaru não se distinguem em sua plenitude (Fig. 09). Entretanto, a quantidade de grupos com distinção da região $\mathrm{N}$ das demais aumentou. Outro aspecto importante dessa terceira análise é a avaliação do efeito de borda, em que a proximidade do topo ou da base da viga pode afetar os resultados dos impactos. Esta análise mostrou que os índices esclerométricos obtidos na posição linha neutra teórica $(\mathrm{N})$ resultaram em valores superiores àqueles obtidos para as outras regiões, assim como nas análises anteriores. Para as vigas C2 e C3 (Fig. 09), nota-se que as diferenciações apresentadas para a força $\mathrm{P}_{10 \%}$ deixou de existir para as intensidades de forças subsequentes. As vigas $\mathrm{C} 1$ e C4 apresentaram homogeneidade nos agrupamentos até a intensidade de força $\mathrm{P}_{30 \%}$. Ao alcançar o carregamento correspondente $\mathrm{P}_{60 \%}$ a viga $\mathrm{C} 1$ apresentou uma diferenciação no agrupamento, entretanto, não sendo possível distinguir as regiões. Para este mesmo estágio de carregamento, com os resultados obtidos para a viga $\mathrm{C} 4$ foi possível distinguir a região $(\mathrm{N})$ das demais.

\section{Vigas da espécie Garapa}

No primeiro cenário (Fig. 10), em quase 50\% dos agrupamentos realizados houve a diferenciação da região $(\mathrm{N})$ das demais regiões e para o nível de $\mathrm{P}_{60 \%}$ houve tal distinção em seis vigas. As vigas G1 e G2 não apresentaram alterações nos agrupamentos devido ao incremento da força de flexão. Por outro lado, para $\mathrm{P}_{60 \%}$ as vigas G3, G4 e G5 apresentaram agrupamento que distingue a região (N) das demais, o que não foi possível para os dois níveis inferiores de força. A viga G6 apresentou tal diferenciação para as intensidades de força $\mathrm{P}_{30 \%}$ e $\mathrm{P}_{60 \%}$, enquanto que a viga G7 apresentou diferenciação para os casos extremos das forças aplicadas $\left(\mathrm{P}_{10 \%}\right.$ e $\left.\mathrm{P}_{60 \%}\right)$.

Os resultados do segundo cenário (Fig. 11) mostram que apenas três agrupamentos foram capazes de distinguir a região neutra das demais regiões, com ocorrência para as vigas G1 (aos níveis de força $\mathrm{P}_{10 \%}$ e $\mathrm{P}_{60 \%}$ ) e G5 (ao nível de força $\mathrm{P}_{60 \%}$ ). Os demais níveis das forças aplicadas às vigas apresentaram agrupamentos que não diferenciam as regiões. 
Veiga e Soriano - Índices esclerométricos avaliados ao longo da altura da seção transversal de vigas de madeira

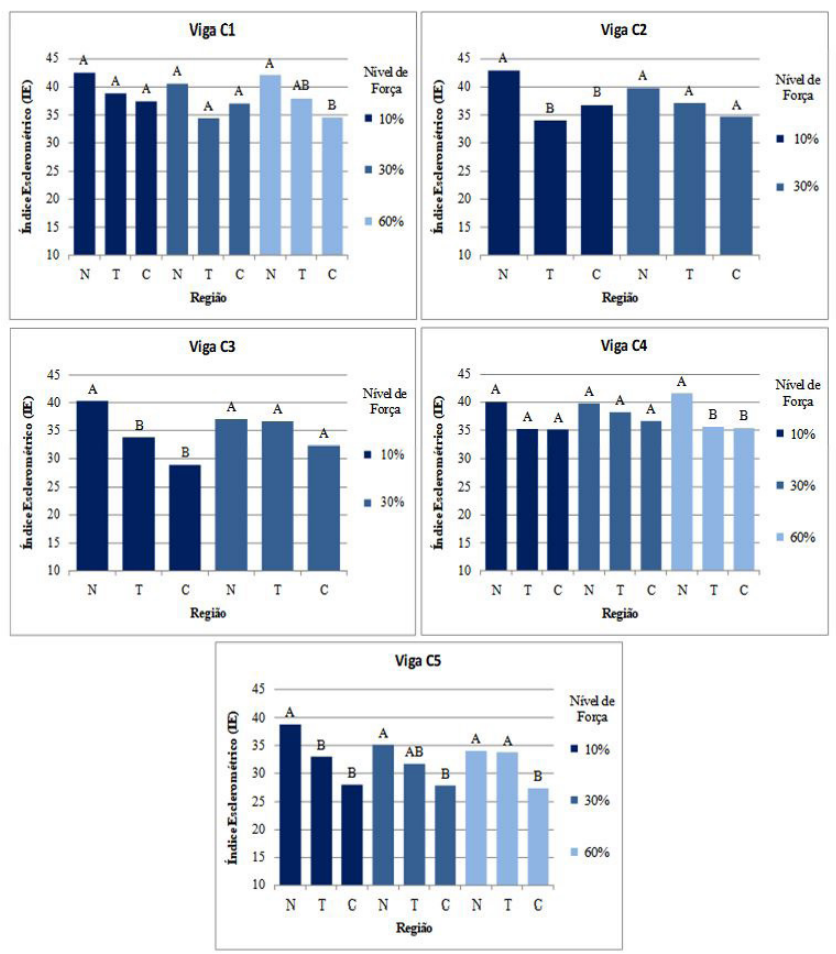

Figura 09. Índices esclerométricos por região de aplicação dos impactos às vigas de cumaru agrupados conforme o terceiro cenário.

Figure 09. Sclerometric indexes by zone of application of impacts to cumaru beams grouped according to the third scenario.

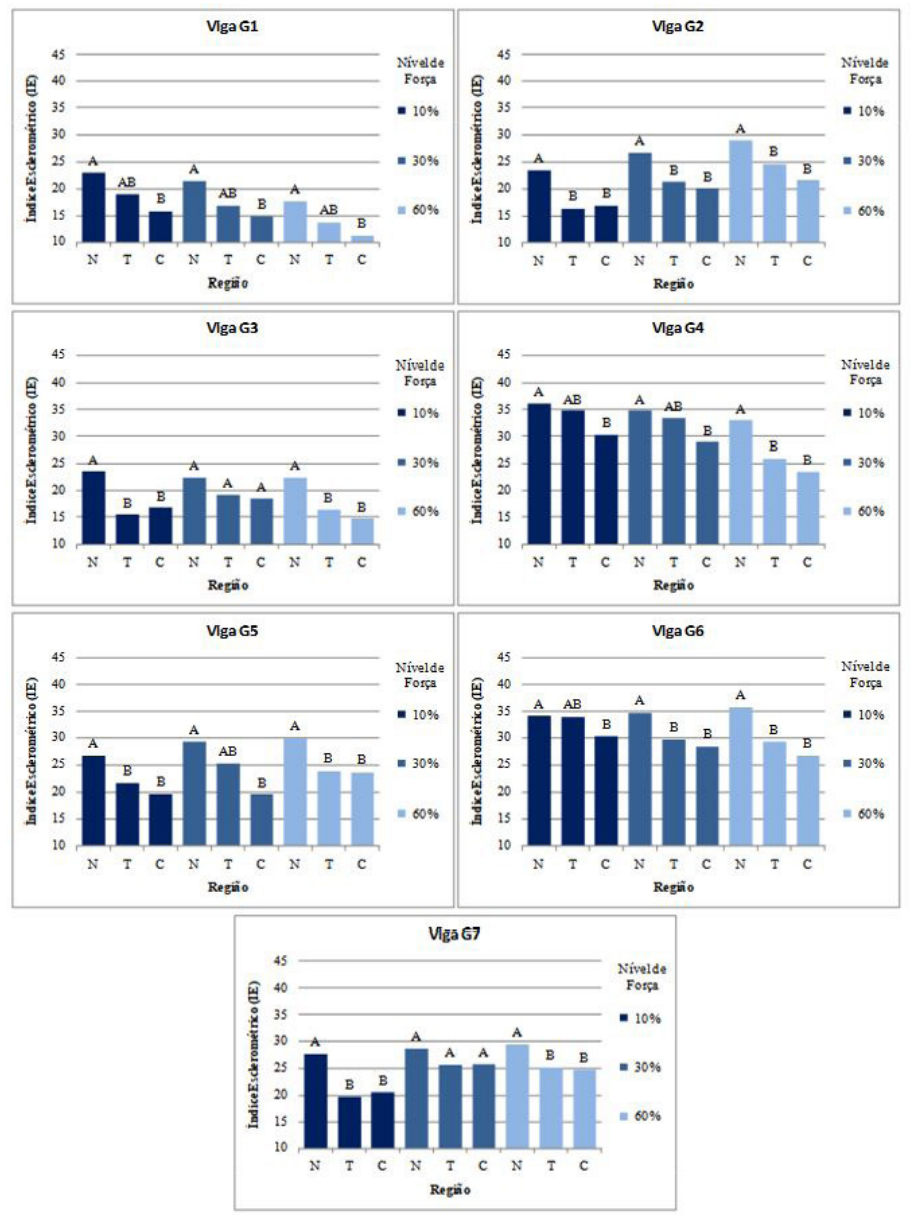

Figura 10. Índices esclerométricos por região de aplicação dos impactos às vigas de garapa agrupados conforme o primeiro cenário.

Figure 10. Sclerometric indexes by zone of application of impacts to garapa beams grouped according to the first scenario. 
Tabela 05. Índices esclerométricos e análise estatística obtidos para as vigas de garapa pelo primeiro cenário.

Table 05. Sclerometric indexes and statistical analysis obtained for garapa beams by the first scenario.

\begin{tabular}{|c|c|c|c|c|c|c|c|c|c|c|}
\hline \multirow{2}{*}{\multicolumn{2}{|c|}{ Viga Análise . }} & \multicolumn{3}{|c|}{$\mathbf{P}_{10 \%}$} & \multicolumn{3}{|c|}{$\mathbf{P}_{30 \%}$} & \multicolumn{3}{|c|}{$\mathbf{P}_{60 \%}$} \\
\hline & & C & $\mathbf{N}$ & $\mathbf{T}$ & C & $\mathbf{N}$ & $\mathbf{T}$ & C & $\mathbf{N}$ & $\mathbf{T}$ \\
\hline \multirow[t]{5}{*}{$\mathrm{G} 1$} & $\mathrm{IE}^{(1)}$ & 15,8 & 23,0 & 18,9 & 14,9 & 21,4 & 16,9 & 10,4 & 17,7 & 13,7 \\
\hline & $\mathrm{DP}^{(2)}$ & 2,9 & 5,1 & 3,3 & 4,2 & 5,5 & 3,6 & 0,5 & 5,1 & 3,6 \\
\hline & $\mathrm{CV}^{(3)}$ & 18,5 & 22,0 & 17,7 & 28,0 & 25,7 & 21,6 & 4,6 & 28,7 & 26,0 \\
\hline & $\mathrm{A}^{(4)}$ & $-0,96$ & 0,09 & 1,10 & 0,79 & 0,01 & 0,41 & 0,85 & 0,09 & 0,26 \\
\hline & $C^{(5)}$ & 0,31 & 0,03 & 1,12 & 0,18 & $-0,61$ & $-0,09$ & $-1,29$ & $-0,76$ & $-0,80$ \\
\hline \multirow[t]{5}{*}{ G2 } & $\mathrm{IE}$ & 16,9 & 23,4 & 16,4 & 20,0 & 26,6 & 21,3 & 21,6 & 29,1 & 24,6 \\
\hline & DP & 4,4 & 4,2 & 5,7 & 3,9 & 3,6 & 3,3 & 2,9 & 3,2 & 3,3 \\
\hline & CV & 25,9 & 18,2 & 34,6 & 19,6 & 13,5 & 15,7 & 13,3 & 11,1 & 13,5 \\
\hline & $A$ & 0,22 & $-0,14$ & 0,31 & $-0,70$ & $-0,62$ & $-0,41$ & $-1,00$ & $-0,54$ & $-0,07$ \\
\hline & C & $-1,79$ & $-0,92$ & $-1,57$ & $-0,12$ & $-0,64$ & $-1,45$ & 0,11 & 0,12 & $-1,51$ \\
\hline \multirow[t]{5}{*}{ G3 } & $\mathrm{IE}$ & 16,7 & 23,5 & 15,4 & 18,5 & 22,4 & 20,2 & 14,6 & 22,4 & 16,4 \\
\hline & DP & 6,2 & 5,8 & 4,4 & 4,2 & 5,4 & 5,5 & 3,5 & 5,6 & 3,9 \\
\hline & CV & 37,0 & 24,5 & 28,6 & 22,5 & 24,1 & 27,4 & 23,6 & 24,8 & 23,6 \\
\hline & $A$ & 0,18 & $-0,37$ & 0,87 & 0,27 & 0,42 & $-0,52$ & 0,42 & $-0,13$ & 0,35 \\
\hline & C & $-1,81$ & $-0,92$ & $-0,07$ & $-1,48$ & 0,13 & $-1,55$ & $-1,14$ & $-0,80$ & $-0,16$ \\
\hline \multirow[t]{5}{*}{ G4 } & $\mathrm{IE}$ & 30,2 & 36,2 & 34,8 & 28,9 & 34,8 & 33,4 & 23,4 & 33,0 & 25,9 \\
\hline & DP & 5,9 & 3,8 & 5,0 & 5,3 & 4,9 & 3,7 & 6,3 & 4,7 & 4,3 \\
\hline & CV & 19,6 & 10,4 & 14,4 & 18,4 & 14,2 & 11,2 & 26,8 & 14,2 & 16,7 \\
\hline & $A$ & $-0,15$ & $-0,27$ & $-1,27$ & $-0,62$ & $-0,01$ & $-0,42$ & $-0,35$ & $-0,59$ & $-0,18$ \\
\hline & C & $-1,96$ & $-0,63$ & 0,33 & $-1,37$ & $-1,23$ & $-0,66$ & $-0,09$ & $-0,19$ & 0,80 \\
\hline \multirow[t]{7}{*}{ G5 } & $\mathrm{IE}$ & 19,6 & 26,7 & 21,7 & 19,6 & 29,3 & 25,3 & 23,6 & 29,9 & 24,0 \\
\hline & DP & 4,0 & 4,3 & 5,1 & 4,4 & 5,2 & 4,1 & 4,2 & 4,0 & 4,5 \\
\hline & CV & 20,5 & 16,3 & 23,4 & 22,6 & 17,7 & 16,2 & 17,8 & 13,5 & 18,9 \\
\hline & $A$ & $-0,39$ & 0,30 & 0,45 & 0,81 & $-0,57$ & 1,12 & $-0,65$ & $-0,13$ & 0,92 \\
\hline & C & $-1,30$ & 0,41 & $-1,84$ & $-1,05$ & $-0,97$ & 0,71 & $-0,76$ & $-0,46$ & $-0,64$ \\
\hline & $\mathrm{IE}$ & 30,3 & 34,1 & 33,9 & 28,4 & 34,7 & 29,7 & 26,6 & 35,7 & 29,3 \\
\hline & DP & 5,2 & 3,3 & 2,5 & 3,5 & 4,3 & 3,4 & 4,4 & 4,4 & 4,9 \\
\hline \multirow[t]{5}{*}{ G6 } & CV & 17,0 & 9,8 & 7,3 & 12,3 & 12,3 & 11,3 & 16,5 & 12,4 & 16,9 \\
\hline & $A$ & $-0,36$ & $-0,16$ & $-0,91$ & $-0,44$ & 0,20 & 0,13 & 0,52 & $-0,92$ & 0,16 \\
\hline & $C$ & $-1,71$ & $-0,99$ & $-0,92$ & 0,93 & $-1,29$ & $-1,02$ & $-1,70$ & 0,66 & $-1,87$ \\
\hline & $\mathrm{IE}$ & 20,5 & 27,7 & 19,8 & 25,8 & 28,7 & 25,6 & 24,7 & 29,4 & 25,1 \\
\hline & DP & 4,1 & 5,1 & 2,9 & 5,3 & 3,1 & 4,8 & 4,9 & 4,0 & 4,0 \\
\hline \multirow[t]{3}{*}{ G7 } & CV & 20,2 & 18,5 & 14,6 & 20,7 & 10,7 & 18,7 & 19,9 & 13,8 & 16,0 \\
\hline & $A$ & 0,10 & $-0,24$ & 0,31 & $-0,49$ & 0,09 & $-0,76$ & 0,11 & 0,09 & 0,63 \\
\hline & C & $-0,76$ & $-0,62$ & $-0,51$ & $-1,04$ & $-1,13$ & $-0,03$ & $-0,61$ & $-1,37$ & 0,77 \\
\hline
\end{tabular}

(I) Índice esclerométrico; (2) Desvio padrão; (3) Coeficiente de variação; (4) Assimetria;(5) Curtose.

(1) Sclerometric index (2) Standard deviation (3) Coefficient of variation (4) Skewness (5) Kurtosis.

Analogamente aos resultados da espécie cumaru, a avaliação dos índices esclerométricos obtidos para as peças de garapa pelo terceiro cenário (Fig. 12) foi aquele que apresentou maior quantidade de agrupamentos (62\%) com diferenciação da região $(\mathrm{N})$ em relação às demais, com diferenciação para as vigas G1 a G6 ao nível da força aplicada $\mathrm{P}_{60 \%}$. Para algumas vigas esse comportamento ocorreu a níveis inferiores de carregamento, como exemplo, das vigas G1 e G2. Entretanto, para outros casos tal diferenciação surgiu com o incremento da força que introduz flexão no elemento estrutural, como são os casos das vigas G4, G5 e G6. A exceção ocorreu apenas para a viga G7, que apresentou diferenciação da região $(\mathrm{N})$ quando submetida ao carregamento correspondente a $\mathrm{P}_{10 \%} \mathrm{e}$, com o incremento da força de flexão essa diferenciação deixou de existir.

Também para essa espécie de madeira a região $(\mathrm{N})$ gerou os maiores índices esclerométricos para os todos os cenários e intensidades de força. Apenas com uma exceção, no caso, a viga G6 com carregamento de intensidade $\mathrm{P}_{10 \%}$. Entretanto, para tal carregamento, os valores referentes às três posições resultaram estatisticamente iguais e, para os incrementos de força subsequentes, os índices esclerométricos da linha central $(\mathrm{N})$ resultaram estatisticamente superiores aos valores das linhas externas $(\mathrm{C} \mathrm{e} \mathrm{T})$. 
Veiga e Soriano - Índices esclerométricos avaliados ao longo da altura da

seção transversal de vigas de madeira

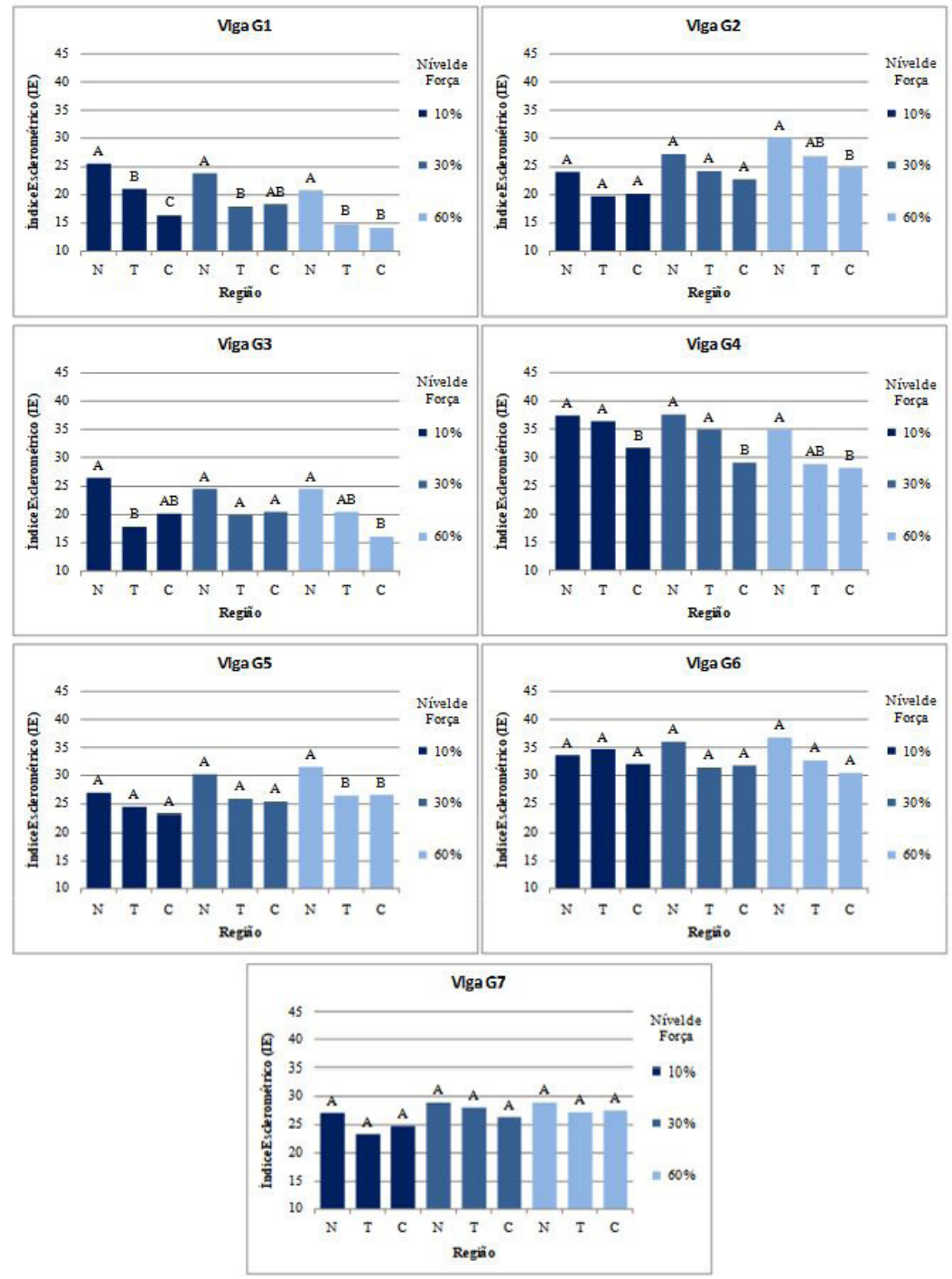

Figura 11. Índices esclerométricos por região de aplicação dos impactos às vigas de garapa agrupados conforme o segundo cenário.

Figure 11. Sclerometric indexes by region of application of impacts to garapa beams grouped according to the second scenario.

Tabela 06. Índices esclerométricos e análise estatística obtidos para as vigas de garapa pelo segundo cenário. Table 06. Sclerometric indexes and statistical analysis obtained for garapa beams by the second scenario.

\begin{tabular}{|c|c|c|c|c|c|c|c|c|c|c|}
\hline \multirow{2}{*}{\multicolumn{2}{|c|}{ Viga Análise . }} & \multicolumn{3}{|c|}{$\mathbf{P}_{10 \%}$} & \multicolumn{3}{|c|}{$\mathbf{P}_{30 \%}$} & \multicolumn{3}{|c|}{$\mathbf{P}_{60 \%}$} \\
\hline & & C & $\mathbf{N}$ & $T$ & C & $\mathbf{N}$ & $\mathbf{T}$ & C & $\mathbf{N}$ & $\mathbf{T}$ \\
\hline \multirow[t]{5}{*}{$\mathrm{G} 1$} & $\mathrm{IE}^{(1)}$ & 16,4 & 25,5 & 21,1 & 18,3 & 23,8 & 17,9 & 14,1 & 20,8 & 14,8 \\
\hline & $\mathrm{DP}^{(2)}$ & 2,8 & 4,2 & 3,9 & 6,4 & 4,0 & 4,4 & 4,7 & 4,4 & 4,4 \\
\hline & $\mathrm{CV}^{(3)}$ & 17,2 & 16,3 & 18,4 & 34,9 & 17,0 & 24,6 & 33,4 & 20,9 & 29,4 \\
\hline & $\mathrm{A}^{(4)}$ & $-0,88$ & 1,34 & 0,04 & 0,67 & 0,22 & 0,60 & 0,79 & 0,05 & 0,70 \\
\hline & $\mathrm{C}^{(5)}$ & $-0,05$ & 1,98 & $-1,53$ & $-0,11$ & $-0,40$ & $-0,62$ & $-0,63$ & $-1,55$ & 0,51 \\
\hline \multirow[t]{5}{*}{ G2 } & IE & 20,2 & 24,1 & 19,7 & 22,8 & 27,2 & 24,3 & 24,8 & 30,1 & 26,9 \\
\hline & DP & 5,5 & 5,5 & 5,3 & 4,6 & 3,0 & 4,8 & 4,6 & 2,4 & 4,1 \\
\hline & $\mathrm{CV}$ & 27,1 & 22,9 & 27,0 & 20,4 & 11,1 & 19,8 & 18,7 & 8,0 & 15,3 \\
\hline & $A$ & $-0,04$ & $-0,36$ & $-0,69$ & $-0,33$ & $-0,78$ & $-0,04$ & 0,03 & $-1,10$ & 0,14 \\
\hline & C & $-1,09$ & $-1,53$ & $-0,56$ & $-0,11$ & $-0,79$ & $-0,98$ & $-0,74$ & 0,75 & $-0,06$ \\
\hline \multirow[t]{5}{*}{ G3 } & IE & 20,1 & 26,4 & 17,8 & 20,4 & 24,4 & 19,8 & 16,1 & 24,4 & 20,4 \\
\hline & DP & 7,3 & 3,8 & 5,3 & 4,2 & 7,6 & 5,0 & 4,3 & 5,4 & 5,6 \\
\hline & CV & 36,4 & 14,2 & 29,7 & 20,6 & 31,3 & 25,2 & 26,9 & 22,2 & 27,6 \\
\hline & $A$ & 0,00 & $-1,00$ & 0,67 & 0,20 & $-0,38$ & $-0,20$ & 0,64 & $-0,23$ & 0,16 \\
\hline & C & $-1,17$ & 1,32 & 0,02 & $-0,02$ & $-0,53$ & $-1,03$ & $-0,47$ & $-1,50$ & $-0,53$ \\
\hline
\end{tabular}

(I) Índice esclerométrico; (2) Desvio padrão; (3) Coeficiente de variação; (4) Assimetria; (5) Curtose.

(I) Sclerometric index (2) Standard deviation (3) Coefficient of variation (4) Skewness (5) Kurtosis. 
Tabela 06. Continuação...

Table 06. Continued...

\begin{tabular}{|c|c|c|c|c|c|c|c|c|c|c|}
\hline \multirow{2}{*}{\multicolumn{2}{|c|}{ Viga Análise . }} & \multicolumn{3}{|c|}{$\mathbf{P}_{10 \%}$} & \multicolumn{3}{|c|}{$\mathbf{P}_{30 \%}$} & \multicolumn{3}{|c|}{$\mathbf{P}_{60 \%}$} \\
\hline & & C & $\mathbf{N}$ & $\mathbf{T}$ & C & $\mathbf{N}$ & $\mathbf{T}$ & C & $\mathbf{N}$ & $\mathbf{T}$ \\
\hline \multirow[t]{5}{*}{ G4 } & IE & 31,7 & 37,4 & 36,3 & 29,0 & 37,6 & 35,0 & 28,2 & 34,9 & 28,7 \\
\hline & DP & 4,8 & 3,4 & 4,4 & 4,5 & 4,6 & 3,9 & 6,9 & 4,2 & 5,6 \\
\hline & CV & 15,3 & 9,0 & 12,0 & 15,5 & 12,4 & 11,1 & 24,5 & 11,9 & 19,4 \\
\hline & $A$ & $-0,64$ & $-0,62$ & $-1,31$ & $-0,56$ & $-0,47$ & $-0,46$ & $-0,57$ & $-0,53$ & $-0,09$ \\
\hline & $\mathrm{C}$ & $-0,58$ & 1,42 & 1,66 & $-0,52$ & $-1,82$ & $-0,53$ & 0,19 & $-0,91$ & $-0,96$ \\
\hline \multirow[t]{7}{*}{ G5 } & IE & 23,3 & 26,9 & 24,4 & 25,4 & 30,2 & 25,9 & 26,5 & 31,6 & 26,4 \\
\hline & DP & 5,5 & 5,6 & 4,8 & 7,3 & 4,9 & 5,0 & 4,7 & 4,6 & 4,8 \\
\hline & CV & 23,7 & 20,8 & 19,6 & 28,6 & 16,3 & 19,1 & 17,9 & 14,5 & 18,3 \\
\hline & A & 0,11 & 0,44 & $-0,58$ & $-0,09$ & $-0,99$ & 0,65 & $-0,58$ & $-0,36$ & 0,11 \\
\hline & C & $-0,21$ & 1,14 & $-1,27$ & $-1,56$ & 1,48 & $-1,05$ & $-0,03$ & $-0,89$ & $-1,32$ \\
\hline & IE & 32,0 & 33,6 & 34,7 & 31,8 & 36,0 & 31,4 & 30,5 & 36,8 & 32,7 \\
\hline & DP & 4,6 & 2,4 & 3,3 & 5,2 & 4,3 & 3,8 & 6,4 & 3,6 & 5,5 \\
\hline \multirow[t]{5}{*}{ G6 } & CV & 14,3 & 7,2 & 9,4 & 16,4 & 12,1 & 12,3 & 20,9 & 9,7 & 17,0 \\
\hline & $A$ & $-0,72$ & $-0,57$ & $-0,33$ & 0,29 & $-0,14$ & 0,41 & 0,17 & $-1,22$ & $-0,29$ \\
\hline & C & $-0,47$ & $-1,77$ & $-0,73$ & $-0,17$ & $-1,86$ & 0,11 & $-1,30$ & 1,74 & $-0,76$ \\
\hline & IE & 24,8 & 27,1 & 23,3 & 26,3 & 28,9 & 28,0 & 27,5 & 28,9 & 27,3 \\
\hline & DP & 5,9 & 6,7 & 5,3 & 3,9 & 2,7 & 4,8 & 4,9 & 4,8 & 4,5 \\
\hline \multirow[t]{3}{*}{ G7 } & CV & 23,7 & 24,9 & 22,6 & 15,0 & 9,2 & 17,0 & 17,8 & 16,7 & 16,5 \\
\hline & $A$ & $-0,11$ & $-0,07$ & 0,61 & $-0,78$ & $-0,83$ & $-0,80$ & $-0,35$ & 0,58 & 0,09 \\
\hline & C & $-0,99$ & $-0,92$ & $-0,25$ & 0,74 & 0,05 & 0,32 & $-0,28$ & $-1,58$ & $-1,15$ \\
\hline
\end{tabular}

(I) Índice esclerométrico; (2) Desvio padrão; (3) Coeficiente de variação; (4) Assimetria; (5) Curtose.

(1) Sclerometric index (2) Standard deviation (3) Coefficient of variation (4) Skewness (5) Kurtosis.

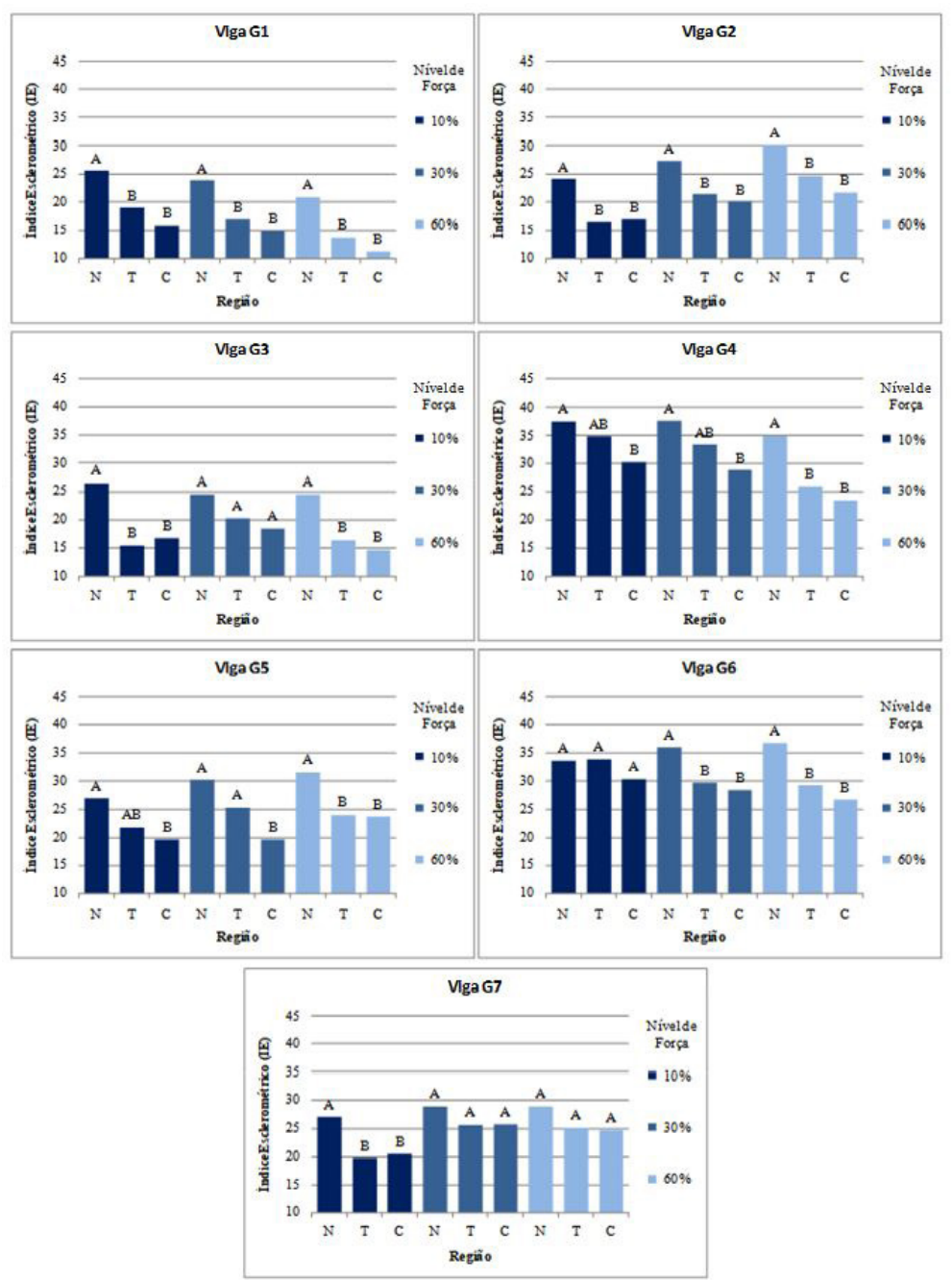

Figura 12. Índices esclerométricos por região de aplicação dos impactos às vigas de garapa agrupados conforme o terceiro cenário.

Figure 12. Sclerometric indexes by region of application of impacts to garapa beams grouped according to the third scenario. 
Veiga e Soriano - Índices esclerométricos avaliados ao longo da altura da

seção transversal de vigas de madeira

A propósito de elaboração de uma metodologia para que o método esclerométrico possa ser aplicado in situ, em que as peças fletidas possam estar sujeitas a tensões normais com intensidades diversas (de zero até um valor último de resistência do material), esses resultados colaboram no sentido de se restringir os impactos na região próxima à meia altura das vigas. Para as vigas das duas espécies sob carregamento de flexão incremental, embora os dados da esclerometria tenham resultado estatisticamente iguais, ou até mesmo não diferenciados, em alguns casos, o índice esclerométrico obtido na região próxima à meia altura da peça $(\mathrm{N})$ resultou numericamente superior àqueles das posições $(\mathrm{C})$ ou $(\mathrm{T})$. A esse comportamento, as exceções foram registradas para a viga C3, no segundo cenário (Tabela 04), bem como para a viga G6 no segundo cenário (Tabela 06), ambos sob o carregamento $\mathrm{P}_{10 \%}$. Entretanto, ainda para essas exceções, a análise estatística revelou para a região $(\mathrm{N})$ índices esclerométricos homogêneos em relação àqueles obtidos em pelo menos uma das regiões externas $(\mathrm{C}$ e $\mathrm{T})$.

$\mathrm{Na}$ hipótese de que todos os agrupamentos tivessem resultado para as posições ( $\mathrm{N}, \mathrm{T}$ e $\mathrm{C}$ ) na configuração A/A/A, ou seja, de homogeneidade para quaisquer intensidades de força, seria possível concluir que tanto o estado de tensões como o efeito de borda não influenciam os resultados do método esclerométrico. Em tal situação, numa inspeção, nas condições desta pesquisa, os impactos poderiam ser aplicados sem restrições em qualquer posição da face lateral da estrutura.

A hipótese de que o estado de tensões normais de flexão influencia os resultados do método esclerométrico pode ser refutada uma vez que as configurações dos agrupamentos apresentaram resultados A/B/B também para as menores intensidades de força e exibiram resultados A/A/A também quando forças de maior magnitude foram aplicadas. Caso a tensão estivesse influenciando os resultados da esclerometria, seria esperado para a menor intensidade de força (A/A/A) e para a maior intensidade (A/B/B). No entanto, há de se considerar a hipótese de que as diferenças encontradas (numéricas e/ou por análise estatística) possam ser atribuídas ao efeito de borda, uma vez que pelo menos para uma das posições mais externas ( $\mathrm{T}$ ou $\mathrm{C}$ ) foram registrados os menores valores dos índices esclerométricos.

\section{CONCLUSÃO}

A avaliação dos resultados dos impactos esclerométricos aplicados ao longo da altura de vigas submetidas ao carregamento de flexão incremental, permitiu concluir que:

- O incremento da força de flexão no elemento estrutural, com consequente aumento das tensões normais de compressão e de tração, pela análise estatística não implicou diretamente nos resultados dos impactos esclerométricos. Com isto, os dois parâmetros (índice esclerométricos e intensidade da força de flexão) não são correlacionáveis.

- Para as vigas de garapa (espécie de menor densidade), na maior parte dos casos houve distinção da linha neutra em relação às regiões de tração e de compressão, principalmente para a intensidade de $60 \%$ da força de ruptura de cada peça.

- Para as vigas de ambas as espécies, na região em que se posiciona a linha neutra resultaram índices esclerométricos superiores àqueles obtidos nas zonas de tração e de compressão.

Ao se avaliar a diferenciação da linha neutra em relação às outras regiões, o terceiro cenário (em que se consideraram os pontos sobre a meia altura da peça e os pontos das linhas mais próximas das bordas) foi o que gerou melhores agrupamentos. Assim, sugere-se que a região da linha neutra seja utilizada para aplicação do ensaio esclerométrico quando este for empregado na estimativa das propriedades da madeira in situ.

\section{AGRADECIMENTOS}

À Coordenação de Aperfeiçoamento de Pessoal de Nível Superior (CAPES) pela concessão da bolsa de doutorado e à Faculdade de Engenharia Agrícola (FEAGRI/UNICAMP) pelo auxílio à pesquisa.

\section{REFERÊNCIAS BIBLIOGRÁFICAS}

ABNT - ASSOCIAÇÃO BRASILEIRA DE NORMAS TÉCNICAS. NBR 7190 Projeto de estruturas de madeira. Rio de Janeiro, 1997. 107 p. 
ANDRÉ, N.; LABBÉ, N.; RIALS, T. G.; KELLEY, S. S. Assessment of wood load condition by near infrared (NIR) spectroscopy. Journal of materials Science, v. 41, n. 7, p. 1879-1886, 2006.

ASTM - AMERICAN SOCIETY FOR TESTING AND MATERIALS. D198-14 - Standard test methods of static tests of lumber in structural sizes. ASTM International, West Conshohocken, PA, 2014. 27 p.

ASTM - AMERICAN SOCIETY FOR TESTING AND MATERIALS. D4761 - Standard test method formechanical properties of lumber and wood-base structural material. Philadelphia, 2013. $11 \mathrm{p}$.

ASTM - AMERICAN SOCIETY FOR TESTING AND MATERIALS. C805 / C805M-13a: Standard test method for rebound number of hardened concrete. ASTM International, West Conshohocken, PA, 2012. 4 p.

BS - BRITISH STANDARDS. BS EN 12504-2: Testing concrete in structures. Non-destructive testing. Determination of rebound number. Londres, 2013. $16 \mathrm{p}$.

FAGGIANO, B.; GRIPPA, M. R.; MARZO, A.; MAZZOLANI, F. M. Combined non-destructive and destructive tests for the mechanical characterization of old structural timber elements. In: INTERNATIONAL CONFERENCE ON ADVANCES IN EXPERIMENTAL STRUCTURAL ENGINEERING. 3., 2009, California. Anais... California: PEER, 2009.

HASEGAWA, M.; YANO, Y.; MATSUMURA, J.; ODA, K. Prospects for within-tree variation of the acoustoelastic behaviors in Japanese cedar. NDT \& E International, v. 49, p. 57-63, 2012.

GONÇALVES, R.; ORMONDE, P. Análise do estado de tensões na madeira utilizando ultra-som. In: CONGRESSO BRASILEIRO DE ENGENHARIA AGRÍCOLA. 34., 2005, Canoas. Anais... Canoas: ULBRA, 2005.

MAHMOUDABADI, E.; AMJAD, U.; KUNDU, T.; SAADATMANESH, H. Effect of applied load on the nondestructive measurement of concrete strength. In: HEALTH MONITORING OF STRUCTURAL AND BIOLOGICAL SYSTEMS, 2014, Bellingham Anais... Bellingham International Society for Optics and Photonics, 2014.

SORIANO, J.; VEIGA, N. S.; MARTINS, I. Z. Wood density estimation using the sclerometric method. European Journal of Wood and Wood Products, v. 73, n. 6, p. 753-758, 2015.

Recebido em: 20/04/2018

Aceito em: 23/10/2018 